\title{
Bioethics in Biotechnology - Bangladesh perspective
}

Dr. R.H. Sarker, Department of Botany, University of Dhaka, Dhaka 1000

Email: rhsarker2000@yahoo.co.uk

Biotechnology consists of a gradient of technology, ranging from long established and widely used techniques of traditional biotechnology through to modern biotechnology based on the application of recombinant DNA technology. The advent of genetically modified crops with pest and disease tolerance, efficient recombinant vaccines and waste-detoxifying bacteria has become a reality. Application of biotechnology has lead to the improvement in lives of people from both developed and developing countries. The economic potential of biotechnology in agriculture, health, energy and environment is well recognized.

On the other hand there also exists apprehension as modern biotechnology may pose certain risks to human health and environment. Bangladesh is relatively new in the field of biotechnology research and development. Many research institutes and universities in the country are currently involved in various plant and microbial biotechnological research. Bangladesh is a signatory of Cartagene Protocol on Biosafety to the Convention on Biological Diversity. In recognition of the importance of ensuring the safe application of biotechnology Bangladesh has developed Biosafety Guidelines as well as a Biosafety Framework. Biotechnological research is being carried out following these biosafety guidelines. In these biosafety documents concerns of ethical issues related to biotechnology has been raised. However, Bangladesh is yet to develop policies on bioethics related to biotechnology. Moreover in general bioethical education is very limited in various phases of science education and research in Bangladesh. 